\title{
Characteristics of field-aligned currents near the auroral acceleration region: FAST observations
}

\author{
W. J. Peria, C. W. Carlson, R. E. Ergun, J. P. McFadden \\ Space Sciences Laboratory, University of California, Berkeley
}

J. Bonnell, R. C. Elphic

Los Alamos National Laboratory, Los Alamos, NM

R. J. Strangeway

Institute of Geophysics and Planetary Physics, University of California, Los Angeles

\begin{abstract}
A pilot statistical study of field-aligned currents (FACs), using an automated FAC-finding technique, has been performed on the magnetometer data from the Fast Auroral Snapshot (FAST) spacecraft. The strategy of this survey is to eliminate all intervals during which the magnetometer data are not consistent with flight through a stationary sheet-like current flowing parallel to the geomagnetic field, and then examine the statistical properties of those data that remain. This survey is sensitive to currents with thicknesses from roughly 10 - $1000 \mathrm{~km}$, over an altitude range of 300- $4300 \mathrm{~km}$, with densities of greater than $0.1 \mu \mathrm{A} / \mathrm{m}^{2}$. We are able to reproduce the familiar statistical location and polarity pattern of large-scale currents, but we emphasize its high variability. The net current (that part which closes along the auroral zone or across the polar cap) comprises not only the large-scale currents, but the more numerous, finely-structured currents as well, implying that the fine structure is, in fact, an integral part of the global current system. FACs show a marked tendency to align themselves with the statistical auroral zone, and an even greater tendency, on each pass, to align themselves with each other, as expected for a population of sheet-like auroral currents. The scale sizes of currents are found to be symmetric with respect to current polarity, but downward currents contain larger spatial gradients of current density, particularly at low values of $K_{\mathrm{p}}$. The likelihood of finding an intense upgoing electron beam within a downward FAC is found to be well correlated with the amount of time its magnetic footpoint has spent in darkness, suggesting suppression of the beams by ionospheric photoelectrons.
\end{abstract}

\section{INTRODUCTION}

The presence of field-aligned current (FAC) is the most fundamental signature of the auroral zone. The presence of FACs is inferred, in this study, from a satellite-borne 3-axis magnetometer, and FACs are seen on essentially every pass. FACs couple electromagnetic energy and stresses from the outer magnetosphere to the ionosphere, and cause significant Ohmic dissipation in the ionosphere. 
The existence of FACs, and their association with aurora, was first proposed by Birkeland [1908], but FACs were not detected by spacecraft magnetometers until much later [Cummings and Dessler, 1967]. Two landmark statistical studies were performed by Zmuda and Armstrong, [1970], and Iijima and Potemra, [1976], using Triad data at $800 \mathrm{~km}$ altitude, and subsequent similar studies include Zanetti et al., [1983]. These studies focused on the location, polarity, intensity, and closure patterns of FACs, and the dependence of these on global geomagnetic conditions. Yamauchi et al., [1998] performed a thorough multi-event study, examining the relationship between large-scale and meso-scale FACs, and how FACs are carried in various local time sectors, as well as reviewing recent FAC studies. For a thorough review of earlier space-based studies of FACs, see Potemra, [1985].

The present study reproduces some of the results of previous space-based studies of FACs, and also performs a high-resolution comparison of FAC magnetometer signatures with energetic electron data, using an automated database technique. This study examines all seasons and local times, and uniformly covers the altitude range within and below the auroral acceleration region, using data selected entirely at random from the first two years of the FAST mission.

\section{Methodology}

All spacecraft studies of FACs to date, including this one, suffer from the same rather serious shortcoming: they cannot directly measure currents! Either the current is inferred, using appropriately scaled time derivatives of the magnetometer data as a proxy for the curl of the local magnetic field, or some part of the current is measured directly (counting the current-carriers), under conditions where the unmeasured (typically low-energy) particles are assumed to be insignificant.

When using a single spacecraft magnetometer to measure currents, there is, of course, the usual space-time ambiguity. But even if it could be ascertained, somehow, that a set of magnetic perturbations was caused by the traversal of timestationary currents, one still would not know if the current sheets were moving or at what rate; thus there would still be systematic uncertainty about how to scale the time derivative of the magnetometer signal into an estimate of the current density. At higher altitudes, Chun and Russell, [1991] have shown, using a two-point measurement (ISEE-1 and 2), that the velocity of current systems can be significant compared to that of orbiting spacecraft, making this systematic uncertainty a serious problem. At the relatively low altitudes visited by FAST, however, the spacecraft velocity $(5-8 \mathrm{~km} / \mathrm{s})$ is typically considerably larger than that of the drift motions of auroral $\operatorname{arcs}(<1 \mathrm{~km} / \mathrm{s})$, which is probably a reasonable 
upper limit for the motions of current systems. The only way to be safe from this uncertainty, of course, is with a multispacecraft measurement.

\subsection{A multiscale automated FAC-finder}

We use an automated FAC-finding routine to catalog FACs. The FAC-finder eliminates all intervals during which FAST is clearly not traversing a sheet of unipolar field-aligned current; what remains are the FACs. The sheer size of the FAST data set makes automated FAC-finding seem necessary. However, automation has the additional advantage that, once the FAC criteria have been established, investigator bias is excluded from the event selection process.

The FAC-finding algorithm is complex and difficult to describe. We will first describe the "multiscale" concept: the idea that since we do not know what size of FACs to search for, we will test for FACs of all sizes. Then, we will describe briefly the information that is recorded about each FAC. Finally, we will explain in detail the FAC criteria: the necessary conditions that an interval of magnetometer data must meet in order to be called a FAC.

For each data point, the FAC criteria are applied to several intervals which are centered on the point in question, and which have a range of different lengths. This procedure is repeated for all data points. The shortest interval which we can sensibly consider contains 3 data points. Since we sample the FAST magnetometer once per second, and since the minimum spacecraft speed is $5 \mathrm{~km} / \mathrm{s}$, there is a lower limit of $15 \mathrm{~km}$ for the thickness of FACs encountered at normal incidence. The longest interval we consider is set by the apparent thickness of the auroral zone on each pass: it is quite variable but typically substantially less than $1000 \mathrm{~km}$. A particular point at a particular interval width will be consistent with the traversal by the spacecraft of either upward FAC, downward FAC, or no FAC at all. Intervals of different lengths, centered on the same point, will not necessarily agree with each other. For example, a long interval about a particular point might be consistent with upward FAC, a shorter interval with downward FAC. When this occurs, the shorter scale is given priority, and in this example the point in question would be said to belong to a downward FAC.

Each such suitable interval produces a single entry in our database. A database entry consists of the location of the FAC, its total size $(\mathrm{km})$, characteristic scale size $(\mathrm{km})$, intensity $(\mathrm{mA} / \mathrm{m})$, peak current density $\left(\mu \mathrm{A} / \mathrm{m}^{2}\right)$, the roughness of its spatial profile $\left(\mu \mathrm{A} / \mathrm{m}^{2} / \mathrm{km}\right)$, its apparent orientation, and several "quality factors". We also compute, for each FAC, the current due to energetic electrons as seen by the FAST electrostatic analyzers (ESAs), and furthermore, an indication of whether or not the interval contains an upgoing electron beam. In this study, we have recorded over 
15,000 FACs, representing roughly $10 \%$ of the FAST data taken (poleward of $\pm 50^{\circ}$ invariant latitude) between 2 October, 1996, and 7 January, 1998.

The following criteria must be met by all the points in an interval of magnetometer data, in order for that interval to be recorded as a FAC.

- The vector difference $\delta \overrightarrow{\mathbf{B}}$ between the measured magnetic field and the IGRF95 model $\overrightarrow{\mathbf{B}}_{0}$ must be within $1^{\circ}$ of the plane perpendicular to $\overrightarrow{\mathbf{B}}_{0}$. (Thus, two components suffice to describe $\delta \overrightarrow{\mathbf{B}}$; these are called "crosstrack" and "along-track", with the obvious meaning.)

- The absolute value of the correlation coefficient between the two components of $\delta \overrightarrow{\mathbf{B}}$ must exceed 0.8 . (This is similar to, though not precisely the same as, demanding that the minimum-to-maximum variance ratio be smaller than a critical value. In practice, the correlation coefficient criterion is somewhat more selective.)

- The angle between the orbit track and the current sheet normal, in the plane perpendicular to $\overrightarrow{\mathbf{B}}_{0}$, must not exceed $60^{\circ}$.

- The current estimated from the time derivatives of $\delta \overrightarrow{\mathbf{B}}$, taking proper account of the apparent sheet orientation, must exceed $0.1 \mu \mathrm{A} / \mathrm{m}^{2}$.

- The sign of the time derivative of the cross-track component of $\delta \overrightarrow{\mathbf{B}}$, which indicates the polarity of the current, must not change.

The application of these criteria, to every data point, and to a variety of interval widths about every data point, yields a 2-dimensional array (the number of data points by the number of intervals sampled), and this must then be collapsed into the start and stop times of FACs. First, at each time point, we find whether current is indicated at any scale. Then, we find the shortest scale at which some amount of current is indicated, and record whether it is upward or downward at that scale. Finally, we find groups of time points where a consistent direction of current is indicated: these are the FACs.

Plate 1 shows an example of the operation of the FACfinder. Plate $1 \mathrm{~A}$ is the "cross-track" magnetometer, the derivative of which is the usual indicator of field-aligned currents. Plate 1B shows the "along-track" magnetometer. These two components of $\delta \overrightarrow{\mathbf{B}}$, and the spacecraft ephemerides, are the only inputs to the FAC-finder.

Plate 1C shows the 2-dimensional output referred to above. The vertical axis shows the width of the windows, about each point, to which the FAC criteria are applied. If the data at a particular time, at a particular scale, are consistent 
with upward current, the corresponding pixel is colored red. Green indicates downward current, and black indicates that the data are inconsistent with the traversal of a sheet-like current. The polarity of each FAC in this example is indicated by the letters "U" (for upward) and "D" (for downward) in Plate 1A. The final output of the FAC-finder is indicated by the green and red vertical lines which span Plates 1A-E. These mark the start (green) and stop (red) times of FACs.

Plates 1D-E are the electron data. Unfortunately, the electron data are not suitable for FAC-finding, since the electron instrument is not always activated, and since the primary current carriers in some FACs are, in fact, unmeasurable thermal electrons. We can, however, compare the FAC-finder output to the electron data whenever it is available. Note how between 2043:35 and 2045:30 there is a net negative deflection of the magnetometer: a net upward field-aligned current. The two upward FACs which comprise it are, however, carried by electrons of rather different character. The downward FAC which interrupts the two upward FACs is apparently carried by the very low energy electrons seen at 0 and $360^{\circ}$ pitch angle. (The green stripe at $90^{\circ}$ is due to an instrumental effect.) A similar configuration (a net current of one polarity interrupted by a FAC of opposite polarity) is seen in the three FACs beginning just after 2041.

The arrow in the third FAC from the left in Plate 1A points at a small fluctuation which might be expected to result in another (very small) FAC. This does not occur however, because this fluctuation is not sufficiently highly correlated with the fluctuation in the along-track signal. Furthermore, the along-track fluctuation at this time is actually larger than the cross-track, indicating that if the fluctuations are due to a current sheet, it is being traversed at very oblique incidence, which makes estimates of current density unreliable. For these reasons, our algorithm rejects the fluctuation indicated by the arrow.

\subsection{The sheet-current assumption}

It is not possible in principle to determine, from a single spacecraft, whether or not a particular magnetic signature is produced by a sheet-like current. In this study, however, we will make an assumption about the geometry of the current systems, since we wish to evaluate the current density within each FAC. We have chosen, as have many other investigators before us, sheet geometry. This choice is not entirely arbitrary, and we attempt to justify it here.

First, a sheet-like geometry is difficult to miss. This is consistent with the ubiquitous and repeatable nature of FACs as viewed from FAST. A relatively small and regular number of currents is encountered on nearly every pass, suggesting that the currents are extended along the auroral zone. Furthermore, the visible aurora, corresponding to regions of up- 
ward current, has a generally sheet-like structure; the radius of curvature of all but the most active arcs is much greater than the thickness of the arcs themselves. This is precisely the sort of "sheet-likeness" required for a reasonably accurate estimate of current density to be made.

Finally, the distribution of apparent current orientations (see Figure 1) appears to contain a substantial sheet-like component. In the spacecraft-centered "cross-track, alongtrack" system, one finds that the most frequently occurring angle $\theta_{c}$ between the sheet direction and the cross-track direction is roughly $20^{\circ}$ (see Figure 1A). The angle $\theta_{c}$ can be transformed to an angle $\theta_{z}$, between the apparent sheet direction and the tangent to the statistical auroral zone [ $\mathrm{Holz}_{-}$ worth and Meng, 1975]. The most frequently occurring value of $\theta_{z}$ is zero (see Figure 1B), and the majority of currents are aligned within $30^{\circ}$ of the statistical auroral zone. On individual passes, however, the sheet orientations are grouped even more tightly than this. Figure $1 \mathrm{C}$ shows the distribution of angles between each current sheet and the median orientation on the pass where each was encountered. The majority of current sheets lie within $15^{\circ}$ of each other on any particular pass of the auroral zone. These tendencies toward alignment give important support to the current-sheet assumption.

The sort of time dependence which might produce the observed magnetic fluctuations (which we and many others interpret as spatial structure) is also strongly constrained by these tendencies toward alignment. Any sort of wave would have to be linearly polarized, and with a preferred orientation aligned along the auroral zone. This is perhaps not too hard to accept, but in the next section of the paper, we will show several results which also constrain the phase of this hypothetical wave relative to the passing of FAST, rendering a temporal interpretation less plausible still.

\section{Statistical Properties of FACs}

\subsection{Regions 1 and 2}

The "Region 1- Region 2" structure of auroral zone FACs [Iijima and Potemra, 1978] can be extracted from our database (see Plate 2), though doing so is not entirely straightforward. The standard picture leads one to expect that, depending on magnetic local time (MLT), any particular pass will yield at most 3 FACs. But fully $90 \%$ of the $\sim 1500$ auroral zone passes (4 per orbit) contained in our database have more than three FACS, and the average number is $\sim 10$. Plate 2 is produced by selecting the most intense upward and most intense downward current from each pass . These opposing pairs are then binned according to MLT. The poleward current of each pair is identified as Region 1, and the equatorward as Region 2. The dominant polarities for Regions 1 and 2 are then found, in each MLT bin. The median 
invariant latitude (ILAT) of each region is then plotted, colored according to polarity, with a latitudinal width equal to the median width of the corresponding FACs. Plate 2 shows the resulting pattern, for two different ranges of $K_{\mathrm{p}}$.

The appearance of a "Region 1- Region 2" pattern under this method is not a foregone conclusion. We are showing that the polarity distribution for the most intense FACs agrees qualitatively with results obtained previously. Our method cannot, of course, reproduce the familiar three overlapping current sheets in the pre-midnight sector, nor at noon. Plate 2 is presented only as a point-of-contact with well-established results. When the differences between the two methods are taken into consideration, our result is consistent with that of [Iijima and Potemra, 1978]; this is a crucial internal consistency check of our methods.

We would, however, like to emphasize that the width of the colored rings in Plate 2 is not a measure of their statistical spread in latitude. Either ring has a typical thickness of only 1 degree, but, at any particular time, may be found as much as $\pm 5^{\circ}$ from its median location; i.e. the statistical spread in latitude of each ring is larger than the difference between the median latitudes of the inner and outer rings. It is sometimes suggested that the instantaneous global current pattern might be parameterized by a single number, such as $K_{\mathrm{p}}$ or IMF $B_{z}$. Figure 2, a scatter plot of the invariant latitude of Region 1 within 1 hour of 9 MLT, demonstrates that $K_{\mathrm{p}}$ cannot be used for this purpose. Although the median latitude does depend on $K_{\mathrm{p}}$, the spread in latitude of Region $1\left(\sim 10^{\circ}\right)$, within each individual $K_{\mathrm{p}}$ bin, is larger than the latitudinal shift of the median across all $K_{\mathrm{p}}$ bins $\left(\sim 5^{\circ}\right)$.

\subsection{The Continuity and Closure of Currents}

The average current density $\overrightarrow{\mathbf{J}_{\mathbf{0}}}$, for a purely parallel current, must scale along a flux-tube as the magnitude of $\overrightarrow{\mathbf{B}}_{0}$. Statistically, this means that the distribution of the ratio $\left|\overrightarrow{\mathbf{J}_{\mathbf{0}}}\right| /\left|\overrightarrow{\mathbf{B}_{0}}\right|$ should show no dependence on altitude.

Since the FAC-finder has the same lower limit for $\left|\overrightarrow{\mathbf{J}_{\mathbf{0}}}\right|$ at all altitudes $\left(0.1 \mu \mathrm{A} / \mathrm{m}^{2}\right)$, it is unable to detect small values of $\left|\overrightarrow{\mathbf{J}_{\mathbf{0}}}\right| /\left|\overrightarrow{\mathbf{B}}_{0}\right|$ at high altitudes, where $\left|\overrightarrow{\mathbf{B}}_{0}\right|$ is small. We therefore cannot examine the entire distribution when making comparisons between altitudes. Figure 3 shows the distribution of $\left|\overrightarrow{\mathbf{J}_{\mathbf{0}}}\right| /\left|\overrightarrow{\mathbf{B}}_{0}\right|>5 \mu \mathrm{A} / \mathrm{m}^{2} /$ Gauss, for altitude bins near apogee, near perigee, and at an intermediate altitude. There is no significant difference between them. The currents therefore appear parallel throughout the covered altitude range, and the low-altitude closure of these FACs must be below $300 \mathrm{~km}$. This point is hardly controversial, but there are few, if any, experimental tests of this basic hypothesis, and the presence of current continuity is a crucial internal consistency check on the FAC database.

The sum of the currents encountered on a given pass is of- 
ten non-zero. This current deficit, or net current, has a definite local time structure, displayed in Figure 4. We produce Figure 4 in three ways. First, in Figure 4A, we use only the Figure 4 intense pairs of FACs used to produce Plate 2. Figure 4B, on the other hand, excludes these most intense FACs, and uses only the remaining weaker, smaller-scale FACs. (By "weaker", we mean that the total current $(\mathrm{mA} / \mathrm{m})$ is less for these FACs.) Finally to produce Figure $4 \mathrm{C}$, we are using all the FACs. The fact that the pattern is present in all 3 cases, but clearest when all FACs are included, indicates that the smaller FACs indeed contribute to the global current system.

Since this net current is not accounted for locally, it presumably returns to the outer magnetosphere after flowing across the polar cap or along the auroral zone. It would also appear that this non-local current closure increases with increasing $K_{\mathrm{p}}$, though this dependence is not statistically significant in Figure 4. Sugiura and Potemra, [1976], reported a similar net current based on their observation of a "level shift" in the approximately east-west component of the magnetic field during traversals of the auroral zone. Their method would not miss any intervals of field-aligned current, but might underestimate the net current, if the predominant sheet orientation differed significantly from the orientation of the single utilized sensor. Our method, on the other hand, risks excluding some intervals which contain current, but properly accounts for the total current of those it detects, utilizing both perpendicular magnetic perturbations. The qualitative agreement in the MLT dependence of the net current, in these two studies, is thus a good consistency check on both.

\subsection{FAC sizes and "roughnesses"}

The largest-scale FACs represent only about $20 \%$ of the currents detected by FAST, and account for roughly half of the total charge transport. Figure 5 shows the distribution of FAC thicknesses. We also define a characteristic scale-size, which is distinct from the thickness of a FAC. This scale-size is intended to quantify the observation that current profiles are not perfectly smooth. The bulk of the current carried by a FAC is typically lumped into sub-regions which are considerably smaller than the FAC itself. We define the scalesize of a FAC as the average size of these sub-regions. The scale-size can then be used to define the spatial inhomogeneity of a FAC's profile. This is done simply by dividing the peak current density by the scale-size, yielding a "typical current gradient" or "roughness". In defining the roughness parameter, one must also map both the current density and the scale-size down to a standard altitude; we chose $110 \mathrm{~km}$.

This particular definition of roughness is intended to avoid some of the difficulties of space-time ambiguity. Both the scale-size and the peak current density, which are used to 
define the roughness, are associated with those parts of a FAC where most of the current is flowing, i.e. with those parts generally accepted (see Plates 2 and 4) to be spatial in nature. The unknowable degree to which we misinterpret temporal structure as spatial is the same for the roughness as it is for those results, in this and past studies.

Generally speaking, FACs with higher intensities $(\mathrm{mA} / \mathrm{m})$ have rougher profiles. This trend is produced both by increasing current densities and by the relative rarity of scalesizes larger than $40 \mathrm{~km}$. Neither the thickness nor the scalesize shows any asymmetry with respect to current polarity. However, downward currents do appear to be somewhat rougher than upward currents, as suggested by some event studies [Carlson et al., 1998; Elphic et al., 1998]. This difference is more pronounced at low values of $K_{\mathrm{p}}, e . g$. the event shown in Figure 1 of Elphic et al., 1998 which has a $K_{\mathrm{p}}$ of $1^{-}$. Figure 6 shows the distribution of roughness with respect to current intensity, for each polarity, for two different ranges of $K_{\mathrm{p}}$.

There is no significant seasonal or solar zenith angle dependence to the roughness of FACs. In terms of MLT, however, we find that FACs within 2.5 hours of noon have a higher degree of roughness (probably contributed to by the complex currents observed equatorward of the "low-latitude cleft current" by Taguchi et el., [1993]). The presence of a definite MLT dependence, combined with the lack of any seasonal or solar zenith angle dependences, suggests that the occurrence of roughness is not related to conditions at the ionospheric footpoint of a FAC, since many different ionospheric conditions occur at the same MLT, as the seasons pass.

\section{THE ASSOCIATION OF FACS WITH UPGOING ELECTRON BEAMS}

It is possible, in principle, to determine the local parallel current density by measuring the flux of electrons at all pitch angles and summing appropriately. Several event studies have already done this on FAST [Carlson et al., 1998; Elphic et al., 1998; McFadden et al., 1998], and shown that the energetic electron flux can sometimes account for all of the current flow in both upward and downward current regions. In downward current regions, the agreement is best when intense upgoing electron beams are present.

Upgoing electron beams are observed preferentially at altitudes above $2000 \mathrm{~km}$, at magnetic local times near midnight, and in the winter. The seasonal and MLT dependencies suggest that a dark ionosphere is needed at the foot of the flux tube in order for a beam to occur. Indeed, as Figure 7 shows, the clearest sorting of beam probability is by the length of time a FAC's footpoint has been in darkness.

\section{Figure 6}

The 5- 6 hour "rise-time" of the beam probability, follow- 
ing sunset, is qualitatively consistent with the characteristic dissociative recombination time for the height-integrated ionospheric electron density [Chamberlin, 1974], ignoring ionospheric convection entirely. This result complements the work of Newell et al., [1996], which discusses the effects of a sunlit ionosphere on auroral electron precipitation.

\section{DISCUSSION}

Our automated study is capable of reproducing past results, as well as going beyond them. The emergence of the familiar Region 1- Region 2 pattern provides reassurance that our FAC-finding algorithm is operating in a sensible way. There is, however, much more going on than the familiar pattern would suggest. The currents used to produce the familiar polarity pattern in Plate 2 comprise about $20 \%$ of the currents in our database, and represent roughly the same amount of total charge transport as the remaining currents combined. The net current pattern seen in Figure 4, on the other hand, is much less clear when the figure is made from only the intense currents of Plate 2. This indicates that the less intense currents are part of the global current system, and are not merely small-scale structures which close locally. Indeed, there is no evidence of substantial local current closure anywhere in our altitude range (see Figure 3).

Downward FACs tend to be "rougher" than upward FACs, and this tendency is more pronounced under quiet geomagnetic conditions $\left(K_{\mathrm{p}}<2\right)$. The significance of this is not presently understood. Hoffman et al.,[1988] reported an increase in the structure of electron precipitation during quiescent auroral periods. The enhanced roughness of downward currents is not simply a manifestation of the structuring of FACs by upgoing electron beams, since it lacks the significant seasonal or solar zenith angle dependence displayed by the beams. There is however, an MLT dependence, with FACs near noon being some 40-50\% rougher, suggesting that the inhomogeneity is determined somehow in the outer magnetosphere, and not in the ionosphere at all.

The ability, provided by FAST, to examine the microscopic particle distribution functions within individual FACs, and to compile statistics on these using an automated procedure, can provide evidence regarding the relative importance of the ionosphere and outer magnetosphere in a given physical process. In this study, the clear sorting of upgoing electron beams, according to the recent illumination history of their ionospheric footpoints, strongly suggests that it is the ionosphere that determines precisely how the return current is to be carried.

Acknowledgments. The authors gratefully acknowledge useful discussions with T. D. Phan and L. M. Peticolas. This research was conducted under NASA grant NAG5-3596. 


\section{REFERENCES}

Birkeland, Kr., The Norwegian Aurora Polaris Expedition 19021903, Volume I, H. Aschehoug and Co., Christiania, 1908.

Carlson, C. W., J. P. McFadden, R. E. Ergun, M. Temerin, W. Peria, F. S. Mozer, D. M. Klumpar, E. G. Shelley, W. K. Peterson, E. Moebius, R. Elphic, R. Strangeway, C. Cattell, and R. Pfaff, FAST observations in the downward auroral current region: Energetic upgoing electron beams, parallel potential drops, and ion heating, Geophys. Res. Lett., 25, p. 2017-2020, 1998.

Chamberlain, J. W., Theory of Planetary Atmospheres: An Introduction to Their Physics and Chemistry, p. 174, Academic, New York, 1978.

Chun, F. K. and C. T. Russell, The evolution of field-aligned currents as a function of substorm phase, J. Geophys. Res., 96, 15801-15810, 1991.

Cummings, W. D. and A. J. Dessler, Field-aligned currents in the magnetosphere, J. Geophys. Res., 72, 1007-1013, 1967

Elphic, R. C. , J. W. Bonnell, R. J. Strangeway, L. Kepko, R. E. Ergun, J. P. McFadden, C. W. Carlson, W. Peria, C. A. Cattell, D. M. Klumpar, F. S. Mozer, M. Temerin, D. Klumpar, E. Shelley, W. Peterson, E. Moebius, L. Kistler, and R. Pfaff, The auroral current circuit and field-aligned currents observed by FAST, Geophys. Res. Lett., 25, p. 2033-2036, 1998.

Hoffman, R. A., M. Sugiura, N. C. Maynard, R. M. Candey, and J. D. Craven, Electrodynamic patterns in the polar region during periods of extreme magnetic quiescence, J. Geophys. Res., 93, 14515-14541, 1988.

Holzworth, R. H. and C. -I. Meng, Mathematical representation of the auroral oval, Geophys. Res. Lett., 2, 377-380, 1975.

Iijima, T. and T. A. Potemra, The amplitude distribution of fieldaligned currents at northern high latitudes observed by TRIAD, J. Geophys. Res., 81, 2165-2174, 1976.

Iijima, T. and T. A. Potemra, Large-scale characteristics of fieldaligned currents associated with substorms, J. Geophys. Res., 83, $599,1978$.

McFadden, J. P., C. W. Carlson, R. E. Ergun, F. S. Mozer, M. Temerin, W. Peria, D. M. Klumpar, E. G. Shelley, W. K. Peterson, E. Moebius, L. Kistler, R. Elphic, R. Strangeway, C. Cattell, and R. Pfaff, Spatial structure and gradients of ion beams observed by FAST, Geophys. Res. Lett., 25, p. 2021-2024, 1998.

Newell, P. T., C.-I. Meng, and K. M. Lyons, Suppression of discrete aurorae by sunlight, Nature, 381, 766, 1996.

Potemra, T. A., Field-aligned (Birkeland) currents, Spa. Sci. Rev., 42, 295-311, 1985.

Sugiura, M. and T. A. Potemra, Net field-aligned currents observed by Triad, J. Geophys. Res., 81, 2155-2164, 1976.

Taguchi, S., M. Sugiura, J. D. Winningham, and J. D. Slavin, Characterization of the IMF By-dependent field-aligned currents in the cleft region based on DE-2 observations, J. Geophys. Res., , 98, 1393-1407, 1993.

Yamauchi, M., R. Lundin, L. Eliasson, S. Ohtani, and J. H. Clemmons, Relationship between large-, meso-, and small-scale fieldaligned currents and their current carriers, in Polar Cap Boundary Phenomena, edited by J. Moen, A. Egeland, and M. Lockwood, pp. 173-188, Kluwer, Dordrecht, The Netherlands, 1998.

Zanetti, L. J., T. A. Potemra, and W. Baumjohann, Ionospheric and Birkeland current distributions inferred from the MAGSAT magnetometer data, J. Geophys. Res., 88, 4875-4884, 1983.

Zmuda, A. J., and J. C. Armstrong, The diurnal flow pattern of field-aligned currents, J. Geophys. Res., 79, 4611-4619, 1974. 
W.J. Peria, Space Sciences Laboratory, UC Berkeley, Berkeley, CA 94720 


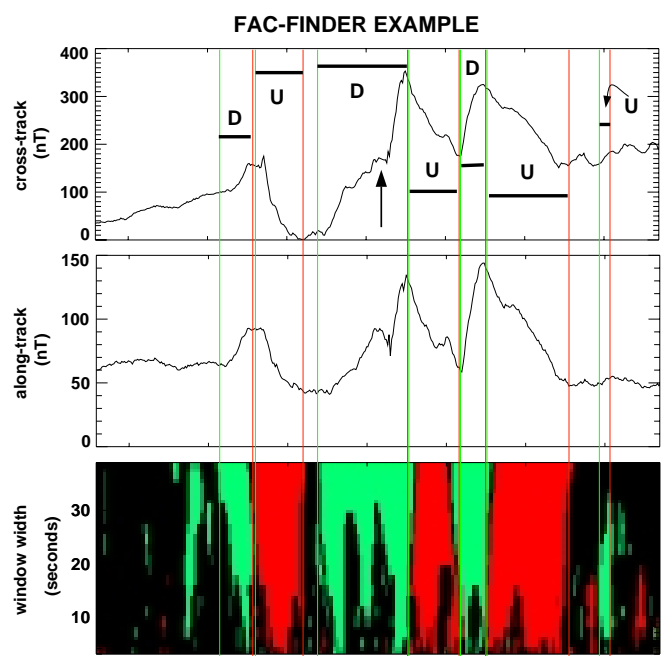

(A)

(B)
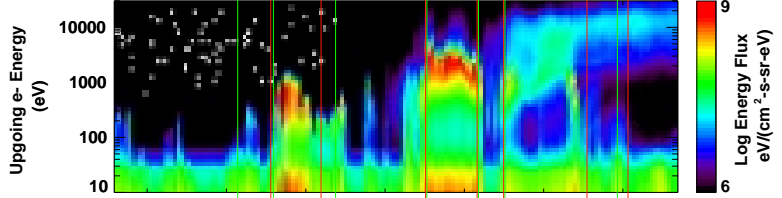

(D)

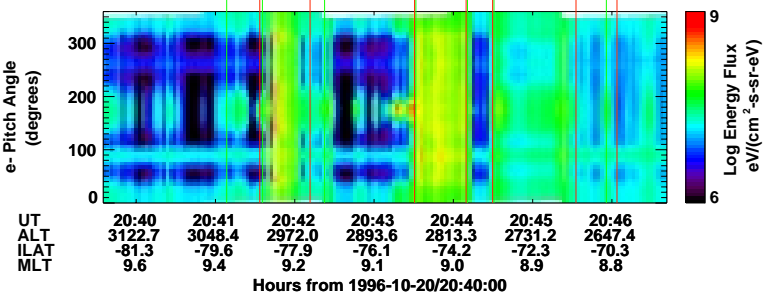

(E)

Plate 1. The output of the FAC-finding algorithm for an auroral zone pass. Also shown are energetic electron data, which provide further confidence in the FAC-finder output.

Plate 1. The output of the FAC-finding algorithm for an auroral zone pass. Also shown are energetic electron data, which provide further confidence in the FAC-finder output. 


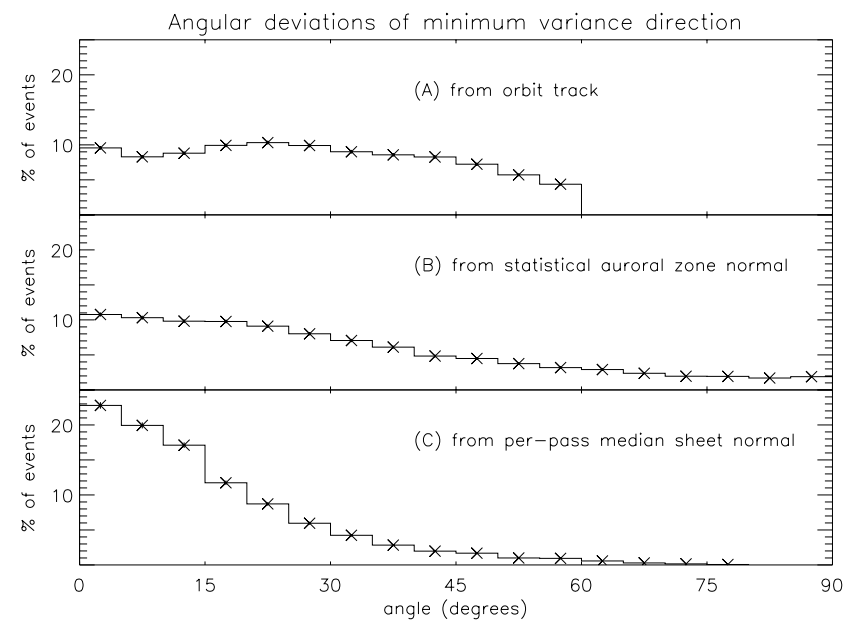

Figure 1. (A) Current sheet normals have a fairly broad distribution with respect to FAST's orbit. (B) The most frequently occurring current sheet normal is perpendicular to the statistical auroral zone. (C) On any particular pass of the auroral zone, the majority of current sheets are aligned to within $15^{\circ}$ of the median orientation.

Figure 1. (A) Current sheet normals have a fairly broad distribution with respect to FAST's orbit. (B) The most frequently occurring current sheet normal is perpendicular to the statistical auroral zone. (C) On any particular pass of the auroral zone, the majority of current sheets are aligned to within $15^{\circ}$ of the median orientation. 

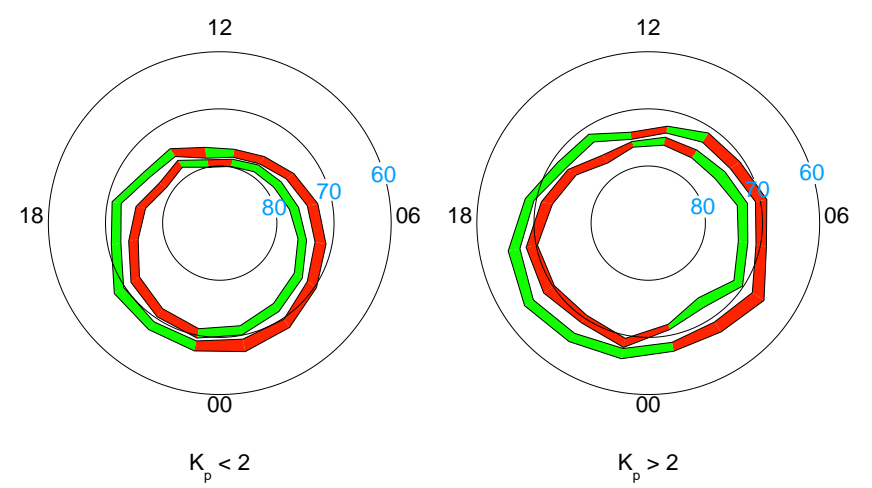

Plate 2. For the two most intense currents from each pass, the median ILAT for each MLT bin is shown. Green indicates predominantly downward currents, while red indicates predominantly upward. The latitudinal width of the bands indicates the median width of the currents in each bin, not their variance in ILAT.

Plate 2. For the two most intense currents from each pass, the median ILAT for each MLT bin is shown. Green indicates predominantly downward currents, while red indicates predominantly upward. The latitudinal width of the bands indicates the median width of the currents in each bin, not their variance in ILAT. 


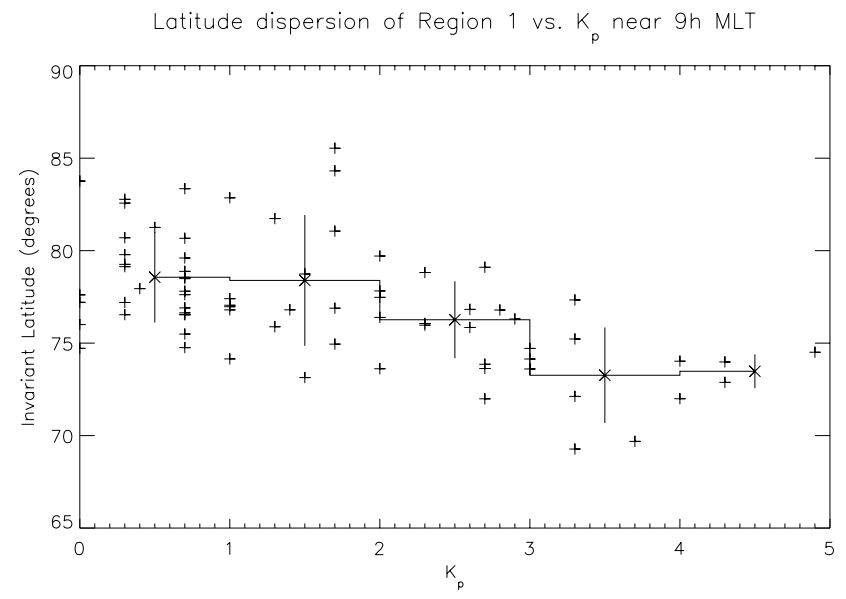

Figure 2. The latitudinal spread of region 1, within 1 hour of 9 MLT, is larger than its $K_{\mathrm{p}}$ dependence, therefore the global current pattern cannot be parameterized by $K_{\mathrm{p}}$.

Figure 2. The latitudinal spread of region 1, within 1 hour of 9 MLT, is larger than its $K_{\mathrm{p}}$ dependence, therefore the global current pattern cannot be parameterized by $K_{\mathrm{p}}$.

Distributions of $J_{0} / B$ at different altitudes

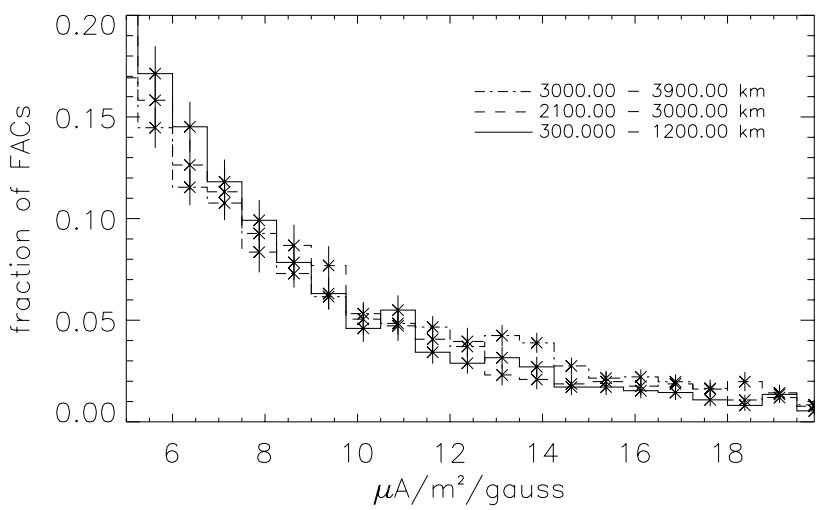

Figure 3. The distribution of $\left|\overrightarrow{\mathbf{J}}_{\mathbf{0}}\right| /\left|\overrightarrow{\mathbf{B}}_{0}\right|$ shows no altitude dependence, indicating that the FACs are, in fact, field-aligned at FAST altitudes.

Figure 3. The distribution of $\left|\overrightarrow{\mathbf{J}_{\mathbf{0}}}\right| /\left|\overrightarrow{\mathbf{B}}_{0}\right|$ shows no altitude dependence, indicating that the FACs are, in fact, fieldaligned at FAST altitudes. 


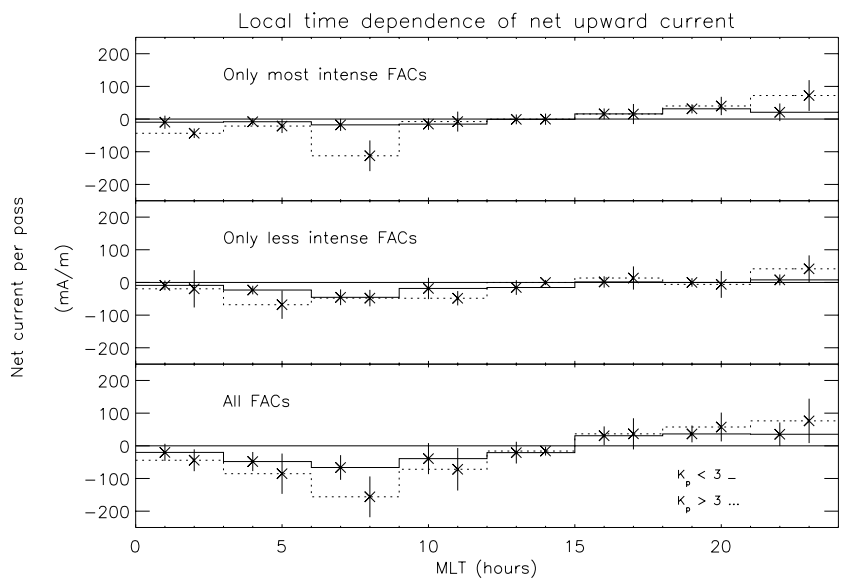

Figure 4. The net current on each pass has a definite local-time dependence, most likely indicative of current closure across the polar cap or along the auroral zone at low altitudes. The pattern is present in the most intense "Region-1 Region-2" FACs, and also in the other less intense FACs, but is seen most clearly when all the detected FACs are considered.

Figure 4. The net current on each pass has a definite local-time dependence, most likely indicative of current closure across the polar cap or along the auroral zone at low altitudes. The pattern is present in the most intense "Region-1 Region-2" FACs, and also in the other less intense FACs, but is seen most clearly when all the detected FACs are considered.

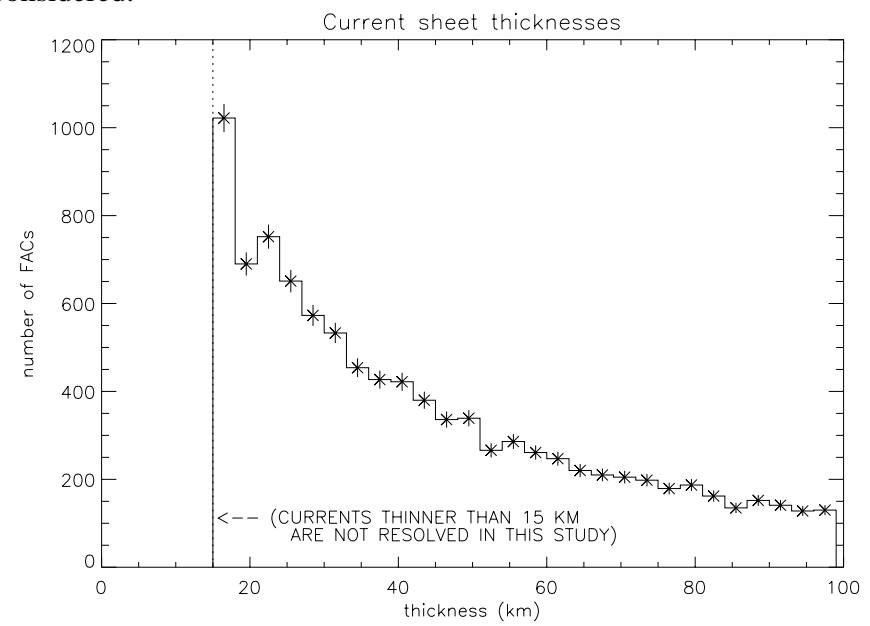

Figure 5. Currents become more numerous at smaller thicknesses. We are not able to detect currents encountered at normal incidence which are thinner than $15 \mathrm{~km}$.

Figure 5. Currents become more numerous at smaller thicknesses. We are not able to detect currents encountered at normal incidence which are thinner than $15 \mathrm{~km}$. 


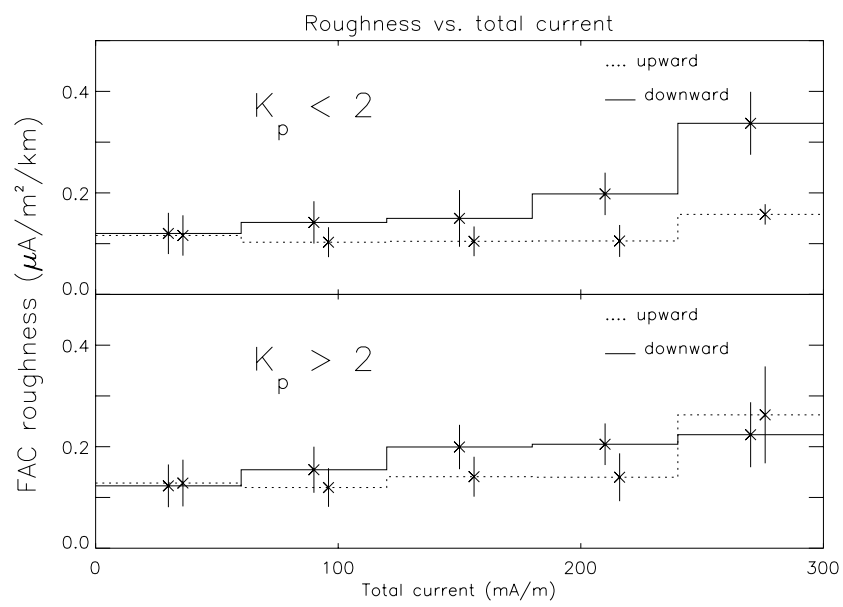

Figure 6. At low $K_{\mathrm{p}}$, the roughness of downward currents depends more strongly on intensity than does the roughness of upward currents.

Figure 6. At low $K_{\mathrm{p}}$, the roughness of downward currents depends more strongly on intensity than does the roughness of upward currents.

\section{Upgoing electron beam probablility}
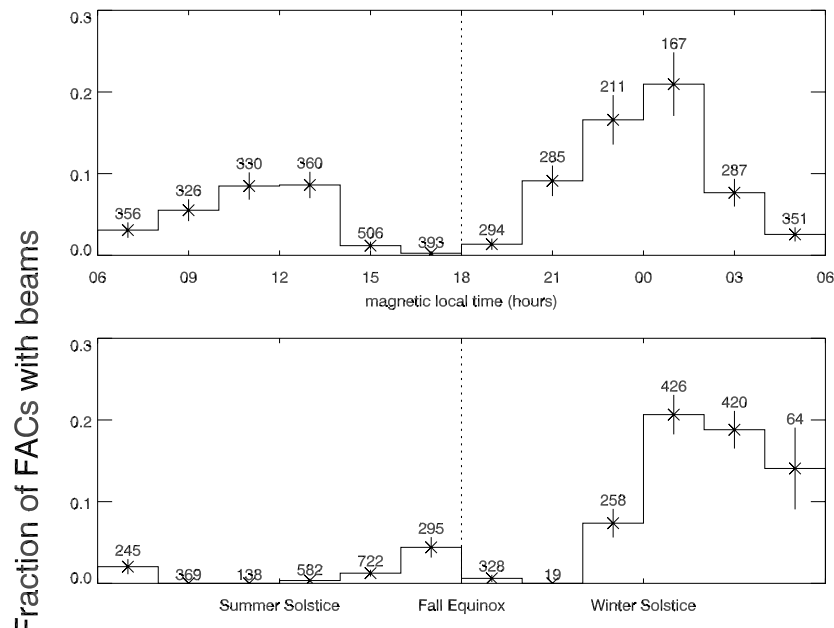

亡ㄴ

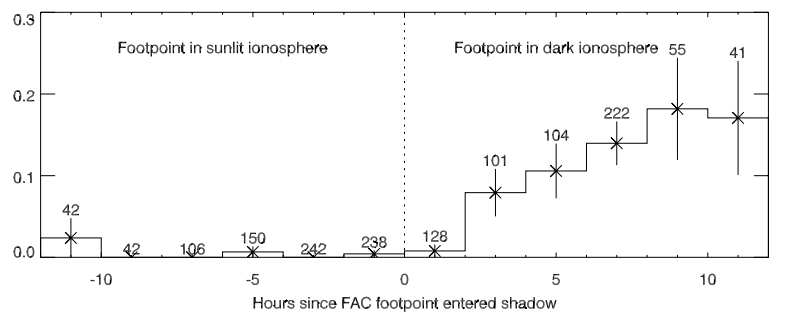


Figure 7. Upgoing electron beams are much more likely to occur on flux tubes which connect to a dark ionosphere. Flux tubes in daylight for 24 hours or more show a beam probability of less than $0.05 \%$. (The numbers indicate how many FACs are in each bin.)

Figure 7. Upgoing electron beams are much more likely to occur on flux tubes which connect to a dark ionosphere. Flux tubes in daylight for 24 hours or more show a beam probability of less than $0.05 \%$. (The numbers indicate how many FACs are in each bin.)

CHARACTERISTICS OF FIELD-ALIGNED CURRENTS

PERIA ET AL.

CHARACTERISTICS OF FIELD-ALIGNED CURRENTS

PERIA ET AL.

CHARACTERISTICS OF FIELD-ALIGNED CURRENTS

PERIA ET AL.

CHARACTERISTICS OF FIELD-ALIGNED CURRENTS

PERIA ET AL.

CHARACTERISTICS OF FIELD-ALIGNED CURRENTS

PERIA ET AL.

CHARACTERISTICS OF FIELD-ALIGNED CURRENTS

PERIA ET AL.

CHARACTERISTICS OF FIELD-ALIGNED CURRENTS

PERIA ET AL.

CHARACTERISTICS OF FIELD-ALIGNED CURRENTS

PERIA ET AL.

CHARACTERISTICS OF FIELD-ALIGNED CURRENTS

PERIA ET AL.

CHARACTERISTICS OF FIELD-ALIGNED CURRENTS

PERIA ET AL.

CHARACTERISTICS OF FIELD-ALIGNED CURRENTS

PERIA ET AL. 
CHARACTERISTICS OF FIELD-ALIGNED CURRENTS

PERIA ET AL.

CHARACTERISTICS OF FIELD-ALIGNED CURRENTS

PERIA ET AL.

CHARACTERISTICS OF FIELD-ALIGNED CURRENTS

PERIA ET AL.

CHARACTERISTICS OF FIELD-ALIGNED CURRENTS

PERIA ET AL.

CHARACTERISTICS OF FIELD-ALIGNED CURRENTS

PERIA ET AL.

CHARACTERISTICS OF FIELD-ALIGNED CURRENTS

PERIA ET AL.

CHARACTERISTICS OF FIELD-ALIGNED CURRENTS

PERIA ET AL.

CHARACTERISTICS OF FIELD-ALIGNED CURRENTS

PERIA ET AL. 